\title{
Correction to: Structure of the Transition Region and the Low Corona from TRACE and SDO Observations Near the Limb
}

\author{
C.E. Alissandrakis ${ }^{1} \cdot$ A. Valentino ${ }^{1}$
}

Published online: 23 October 2019

(C) Springer Nature B.V. 2019

\section{Correction to: Solar Phys. (2019) 294: 96 \\ https://doi.org/10.1007/s11207-019-1486-7}

In computing the height with respect to the $\tau_{5000}=1$ level, the height difference between the white light limb and that level (see first paragraph of Section 3.3) was subtracted rather than added to the values in Table 5. This affects Sections 3.3 and 3.4, Figures 14 and 15, as well as Table 5. The correct versions are given below.
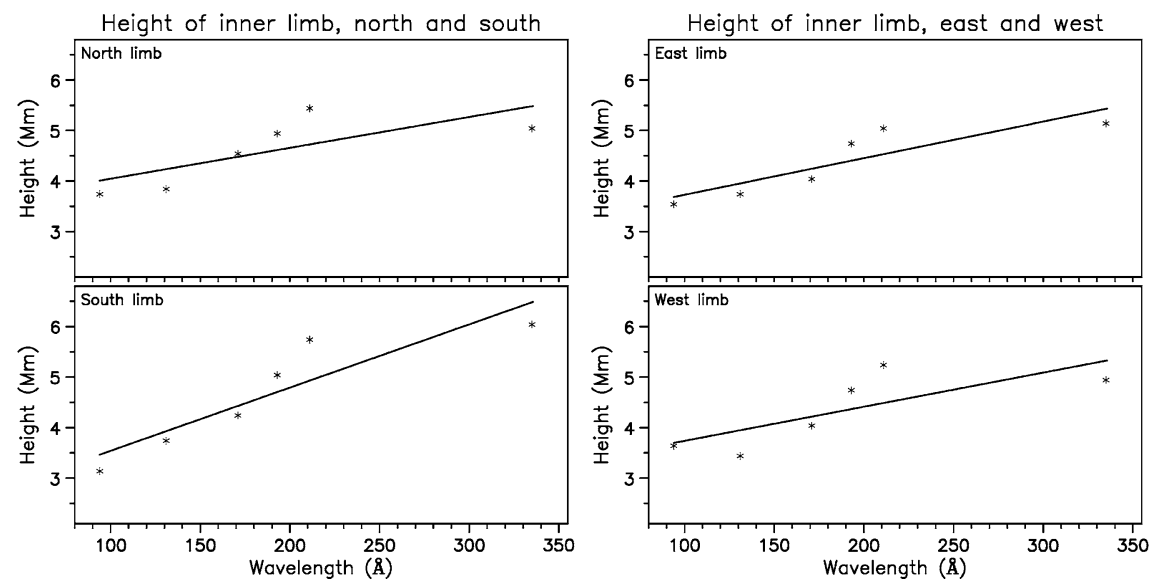

Figure 14 The height of the inner limb with respect to the $\tau_{5000}=1$ level for the AIA TR channels as a function of wavelength. The straight lines show the result of a linear regression.

The online version of the original article can be found under https://doi.org/10.1007/s11207-019-1486-7.

\section{C.E. Alissandrakis} calissan@cc.uoi.gr

1 Section of Astro-Geophysics, Department of Physics, University of Ioannina, 45110 Ioannina, Greece 
Figure 15 Computed column density of neutral hydrogen as a function of height above the $\tau_{5000}=1$ level. Black is for the north polar region, red for the south. Both sets were used for the computation of the regression line.

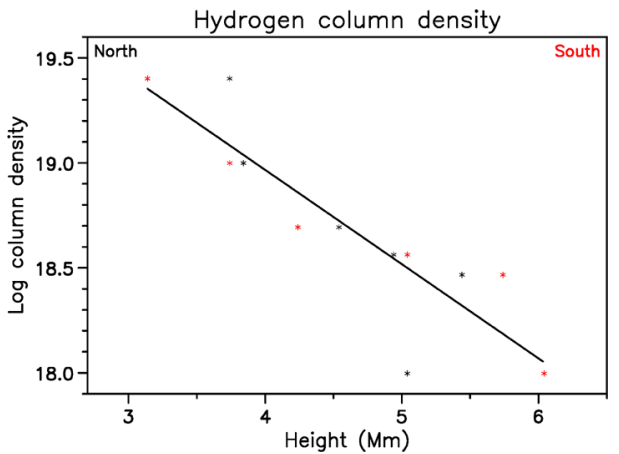

Table 5 Hydrogen density at $4000 \mathrm{~km}$ and scale height.

\begin{tabular}{lllll}
\hline $\begin{array}{l}N_{c} \\
\mathrm{~cm}^{-2}\end{array}$ & $\begin{array}{l}N_{\mathrm{HI}} \\
\mathrm{cm}^{-3}\end{array}$ & $\begin{array}{l}N_{\mathrm{H}} \\
\mathrm{cm}^{-3}\end{array}$ & $\begin{array}{l}\text { Scale } \\
\mathrm{Mm}\end{array}$ & Reference \\
\hline $6.25 \times 10^{18}$ & $\begin{array}{l}9.42 \times 10^{8} \\
-\end{array}$ & $1.88 \times 10^{9}$ & 0.97 & This work \\
- & $1.36 \times 10^{10}$ & - & 1.25 & Daw, Deluca, and Golub (1995) \\
- & - & $0.55 \times 10^{9}$ & 4.1 & Avrett and Loeser (2008) \\
\hline
\end{tabular}

In addition to the above:

In the abstract, the phrase "We estimated a height of $(2300 \pm 500) \mathrm{km}$ for the base of the transition region" should be replaced by "We estimated a height of $(3000 \pm 500) \mathrm{km}$ for the base of the transition region."

In the last paragraph of page 16, (Section 3.3) the phrase "at heights of 2.5 and $5 \mathrm{Mm}$ above the $\tau_{5000}=1$ level respectively (Figure 15)" should read "at heights of 3.2 and $5.7 \mathrm{Mm}$ above the $\tau_{5000}=1$ level respectively (Figure 15)."

The last phrase in Section 3.3, "our density value is about twice that of the Avrett et al. (2015) model, with a considerably smaller scale height" should be replaced by "our density value is similar to that of the Avrett et al. (2015) model, with a considerably smaller scale height".

The first paragraph of Section 3.4 should be replaced by: "The presence of spicules does not allow a direct measurement to be made of the average height of the top of the homogeneous chromosphere. An upper limit can be deduced from the minimum height of the inner limb at $94 \AA$, which is about $3700 \mathrm{~km}$ above the $\tau_{5000}=1$ level. A better estimate can be obtained from the extrapolation of the regression line of the average inner limb height versus wavelength plot (Figure 14) to $\lambda=0$, where there is no chromospheric absorption. This gives a height of $(3000 \pm 500) \mathrm{km}$, a range which is above the value of $2140 \mathrm{~km}$ used by Avrett and Loeser (2008)."

The fourth paragraph of Section 4 should be replaced by: "Assuming that the optical depth of the chromospheric absorption in the TR bands is unity at the height of the inner limb, and attributing the absorption to neutral hydrogen and helium, we computed the neutral hydrogen column density as a function of height; we obtained values between $\sim 2.2 \times 10^{19}$ and $\sim 1.6 \times 10^{18} \mathrm{~cm}^{-2}$, at heights between 3.2 and $5.7 \mathrm{Mm}$ above the $\tau_{5000}=1$ level. From these results and for a homogeneous chromosphere, we computed a neutral hydrogen density of $9.4 \times 10^{8} \mathrm{~cm}^{-3}$ at a height of $4 \mathrm{Mm}$ and a scale height of $0.97 \mathrm{Mm}$." 
Finally, in the caption of Figure 4, the phrase "The planet shows up as a dark disk in the NW quadrant" should be replaced by "The planet shows up as a dark disk in the NE quadrant". Moreover, the units in Equation 6 should be $\mathrm{cm}$ rather than Mm.

Publisher's Note Springer Nature remains neutral with regard to jurisdictional claims in published maps and institutional affiliations. 